\title{
Design and Realization of a Certain Type of Radio Power Amplify Circuit Board Detecting and Teaching Platform
}

\author{
Zhang Wei, ${ }^{1, a}$, Shao Zhichao, a , Cheng Huihua ${ }^{1, a}$, Zhou Quanzhi ${ }^{1, a}$, \\ Lou XiaoFang ${ }^{1, a}$, Xiao Hang ${ }^{1, a}$ \\ ${ }^{1}$ Wuhan Mechanical Technology College, WuHan, HuBei, 430075, China \\ ashaozhichao@sina.com
}

Keywords: Power Amplify Circuit Board; Detecting and Teaching Platform; adapter; Teaching Software

\begin{abstract}
A certain type of command and control system detecting and teaching platform is designed in order to realize clear display of the operation, implement of the capability teaching, detecting and fault diagnoses conveniently. This thesis which is a part of the program introduces the design and realization of a certain type of radio power amplify circuit board detecting and teaching platform. The platform consists of adapter and Teaching Software Experiments indicate that the platform is reliable in the operation, can improve the efficiency of circuit board teaching and provide a method for circuit board detecting and fault diagnoses.
\end{abstract}

\section{Introduction}

A certain type of radio is a high-tech electronic information equipment with the characteristics of complex structures and expensive cost, which is widely used in our army's various command and control systems. As the most important wireless communications equipment in the command and control system, the quality of working conditions of the radio directly determines operational performance of the command and control system. Therefore, there is a growing concern for the training of staff who are responsible for the operation and use and maintenance support of the radio.

At present, there is a lot of inconvenience in the teaching of working principles of the radio, especially circuit working principles. Only when the radio is taken apart can it show its internal structure. Due to the internal circuit boards of the radio are designed in sub-block and packaged respectively, and the circuit boards must be encapsulated when working with charge, there is no way to implement the live signal detection and analysis with electricity to key test points of the circuit board of the radio. To solve this problem, a detecting and teaching platform of a certain type of command system is developed which can solve the difficulties described above, and implement the teaching of the circuit operating principle of a certain type of radio printed circuit board (PCB) level and fault diagnosis visually.

\section{Platform Introduction}

The hardware components of a certain type command system detecting and teaching platform can exert excitation signal to the circuit board when the printed circuit board waiting be detected is from the individual equipment. The platform creates the working environment, which ensures the circuit board can work in normal state, measures actually key points signal of the circuit board by universal testing equipments, and also by the host computer to display the working principle of PCB, signal flow, as well as the theoretical value of key points signal. Through the combination of hardware and software, the platform developed a variety of adapters and teaching software for different types of printed circuit boards. Teach platform has the following functions.

- The teaching platform can demonstrate all the functional requirements and operating principles of the key PCB and the structure of owned equipment. 
- Teach platform provides the corresponding adaptation interfaces to the PCB hardware, and carry out fault detection and location on the circuit board.

- The teaching platform is able to display the signal flow of all important PCB, and the theory signal OF PCB key points and the signal waveforms measured actually.

- The teaching platform can display vividly the whole process of fault diagnosis of PCB.

\section{Introduction of the Amplifier Board}

The main function of the radio amplifier board is to zoom the excitation signal amplification sent by RF Ring to $26 \mathrm{dBm}$ into $4.5 \mathrm{~W}$ and then output it. And a receive switch is provided. The amplifier board contains a receiver channel, a transmitter channel and a receive switch. The output of receiver channel will be sent to the tuner 's high-level input; the input of transmitter channel is from the RF ring output. The transmitter channel consists of an RF amplifier, three band-pass filter with different frequency ranges and two corresponding sets of electronic switches, the level of power switch, power control and protection, as well as power detection. The Amplifier board schematic framework diagram is shown in Figure 1.

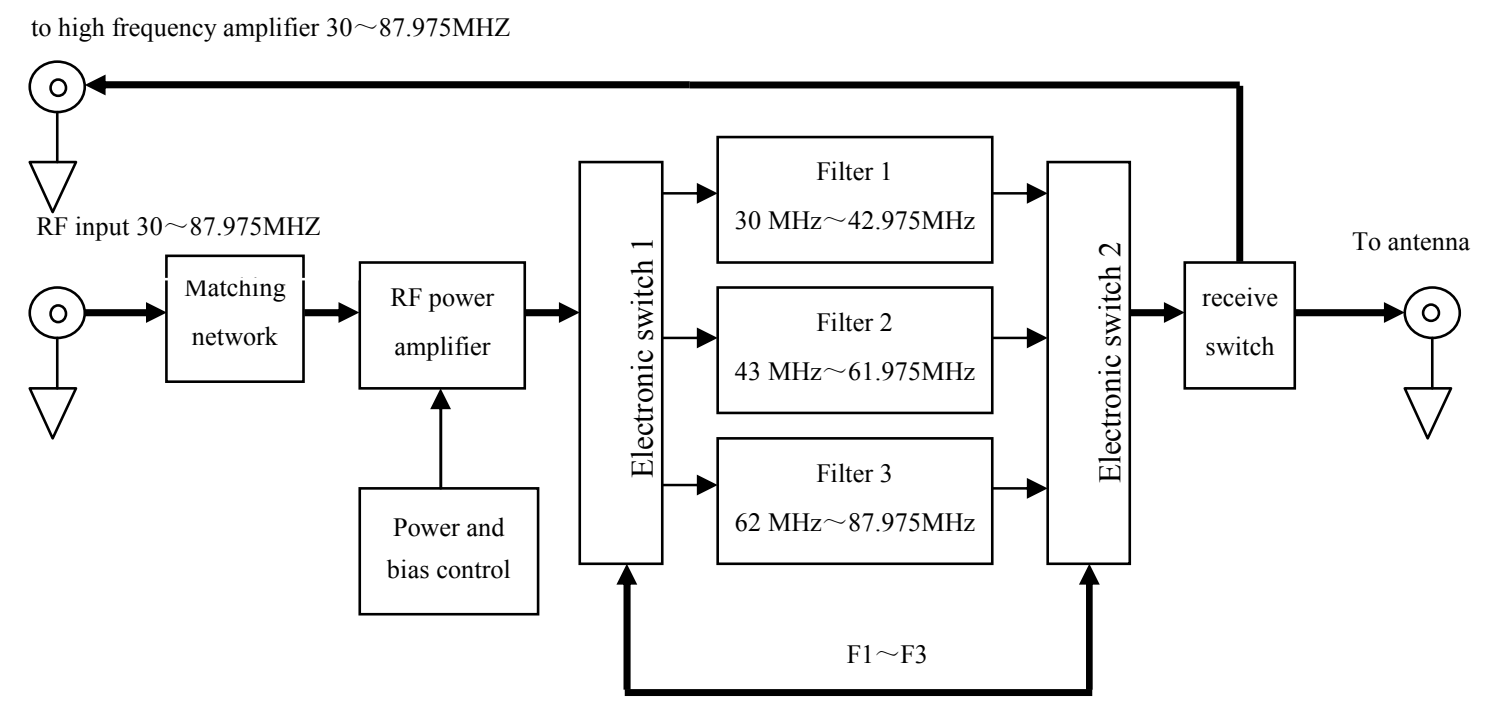

Figure 1 The Amplifier board schematic framework diagram

\section{Platform design and implementation}

The detecting and teaching platform is composed of undetected PCB, PCB adapter, the host computer, as well as general testing equipment. The structure of the command system PCB general detection teaching platform is shown in Figure 2.

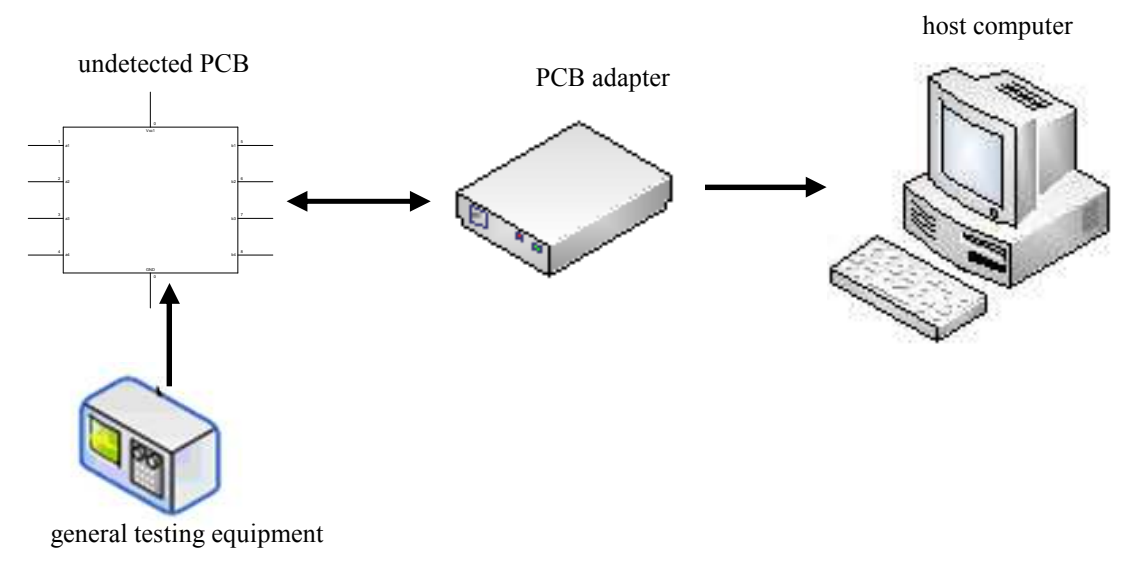

Figure 2 The structure of a certain command system PCB general detection teaching platform 
The platform will develop corresponding PCB adapters for all types PCB. The adapter, by receiving commands from the host computer serial port, provides the required power input and excitation input to the undetected PCB, and then after receiving the signal output from the undetected PCB to sent the output signal to the host computer through the serial port eventually. Running on the host computer, the detecting and teaching software can control the adapter to generate different excitation signals to the undetected PCB; after the host computer receives the feedback signal from the adapter, the test results will be displayed on the monitor in the form of graph. At the same time, the software system of the detecting and teaching platform is able to demonstrate all the key functional requirements, operating principles of the PCB and the structure of the equipments which they belong to. The general testing equipment, which consists of multimeters, oscilloscopes, logic analyzers and etc., is used to test the actual signals of the critical detection point of the PCB. Besides, the platform is also equipped with a DC power supply, excitation sources like the signal generator.

- Hardware Design of the Platform

The platform hardware provides the circuit boards with corresponding adapters, in accordance with the specific circumstances of the PCB, different adapters will be developed to meet the different working states of the PCB. Through receiving the commands sent by the host computer serial port, the adapter sets out to adjust the working condition, provides a variety of excitation signal to the undetected PCB, and at the same time sends commands to the host computer through the serial port. After the adapter controls the working status of the circuit board and the PCB works properly, the same test results(theoretical value) with the host computer software will be achieved by using the generic detection equipment to test the measured values of the key points of the circuit board so as to achieve the detecting and teaching function of the PCB. A certain type of radio amplifier board adapter design diagram is shown in Figure 3.

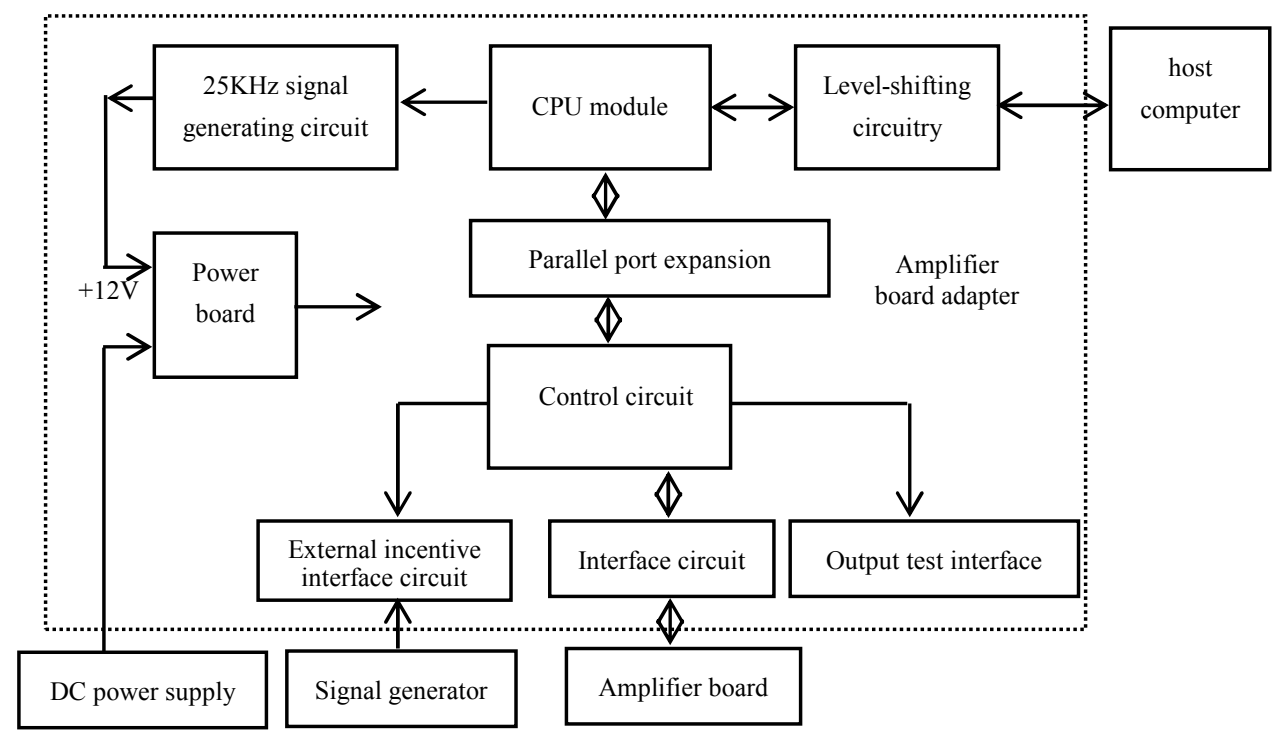

Figure 3 Amplifier board adapter structure

- Software Design of the Platform

The PCB detecting and teaching software is the software proportion of the host computer in the detecting and teaching system, which is used to display the structure of the PCB, operating principles, schematics, circuit board and the key point signal theory waveform. After selecting the PCB with specific model of equipment, the detecting and teaching software will send instructions to the PCB through the host computer serial port adapter. And then the adapter will generate various excitation signals required in the running of PCB to further controls and adjusts the working status of the circuit board. Next, through testing the key points of the circuit board by general detection device, the detecting and teaching software can make a comparative study with theoretical signal of the host computer software, to achieve the teaching function of the PCB. 
The signal detecting function of the circuit critical points in the detecting and teaching software is realized through the following programs: increase the critical points in the circuit diagram, corresponding theoretical value waveform is pre-stored in the corresponding critical points, then click the key point and the teaching software interface can display the pre-stored signal waveform on the host computer.

Each adapter corresponds to one or more PCB, each PCB corresponds to its the circuit diagram and the main interface of teaching software which consists of PCB digital real photos, digital schematic of the PCB and PCB critical detection point signal theoretical waveforms. The platform developing software chooses $\mathrm{VB}$, the teaching software operating results interface is shown in Figure 4, the software interface can display the circuit schematics, circuit board photos, the specific location of critical detection points in the circuit board and the detection point signal waveform simultaneously. Users can choose one from the adapters in using, and then select one PCB from the specific adapters. The teaching software communicates with PCB adapter via the host computer serial port, then sends commands to the adapter through the serial port. The commands are not only the basis of the adapter to generate the corresponding excitation signal, but also can receive the command sent back through the serial port adapter.

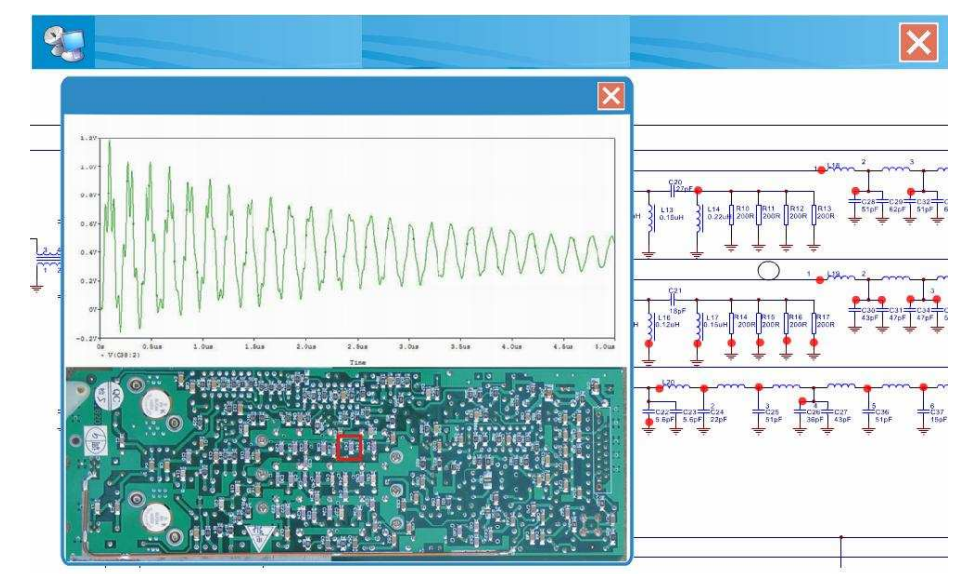

Figure 4 Interface diagram of the detecting and teaching software detection

\section{Conclusions}

By using the adapter the detecting and teaching platform provide a work environment for the PCB, which can not only make the radio circuit board work properly without from the radio itself, but also show perfectly the structure of radio internal circuit board and its working principle, at the same time it completes detecting, teaching, the task of testing and fault diagnosis of the radio the circuit board. It greatly facilitates the mounting of teaching and fault detection and maintenance, overcomes the difficulties of non-intuitive and unpicturesque display in traditional mounting instruction mode which eliminates the unnecessary work of complex disassembly and test equipment connections. Through software and hardware environmental testing, the platform runs stably and reliably, which can greatly improve the efficiency and level of implementation in teaching, as well as provide a convenience for the actual test and maintenance.

At present, this detecting and teaching platform has been used in practical teaching. After a period of using, it is reported that the platform runs well, easy to learn and use. But there are two problems : First, when the circuit board is in normal working in the radio internal, it is included in a shielded cavity, which forms an inhibition for the RF signal, but when the working environment of the radio circuit board is provided through the adapter, there is no the external environment of the shielding cavity, which results in a slight change of key RF signal of part detection points in circuit board, but it does not affect the normal working of the entire board; Second, the length of the radio circuit board $\mathrm{RF}$ cable is changed in the adapter design process, part of the RF signals attenuate somewhat in the 
detection, but it does not affect the normal working of radio circuit board under tested and the detecting and teaching platform. Further work to be done in this paper is to solve the above two problems.

\section{References}

[1] Liu Cun, Modern Detection Techniques [M] Beijing: Mechanical Industry Press, 2005, pp.65-69.

[2] Zhou Yuwei, Automatic Detection Technology [M] Wuhan: Huazhong University of Science Press ,1995, pp.112 -116.

[3] Houbo Heng, Libo Cheng, 16 Microcomputer Principle and Interface Technology [M]. Xi'an: Electronic Science and Technology University Press ,1996, pp.79 -83.

[4] Mei Jinguo, Yang Zhiping, IC Application Design [M]. Xinyang: The First Aeronautical College Press ,2000, pp.124 -127.

[5] Yu Mengchang, Fundamentals of Digital Electronics Concise Guide [M] Beijing: Higher Education Press ,1999, pp.98 -104. 